\title{
Cultural weight dragging at Asian giants' feet
}

\section{Scientific progress is impeded by a tradition of conformity and respect for authority.}

Sir - Reading the Commentary "Cultural reflections" by $\mathrm{Mu}-\mathrm{ming} \mathrm{Poo}$, on the subject of Chinese science (Nature 428, 204; 2004), I was struck by the similarities between science in China and in India.

Poo concludes that mediocrity in Chinese science should be attributed to cultural factors such as conformity and respect for authority, rather than to pure economics. India, like other Asian countries, is at a crossroads. It is time for these countries to free themselves from the burden of their past and to develop a modern structural framework for fostering original scientific research.

Both India and China are emerging into modernity. Each has a booming urban economy (with vast problems in the rural areas) and India's gross domestic product (GDP) has suddenly increased in recent years, thanks to liberalization and private entrepreneurship. India's overall funding in basic research has also improved, and now it is $1.2 \%$ of GDP.

Yet although India has a considerable number of scientists, it cannot be counted as a major world player in basic sciences, notwithstanding its performance in space and nuclear technologies.

Not a single recent issue of Current Science, India's premier science journal, has appeared without at least one critique of India's performance in basic sciences, for example concluding that India and China face very similar problems (P. Balaram Curr. Sci. 86, 755-756; 2004).

Twenty years ago, John Maddox wrote, about science in India, "Among developing nations, India has by far the best chance of succeeding" (Nature 308, 581-584; 1984). However, he also wrote, "India has set ambitious goals for science and technology - self-reliance and the relief of poverty. Some great things have been accomplished, but much effort is frustrated."

The Pakistani physicist and Nobel laureate Abdus Salam has been quoted as saying during a visit to India in 1981, "Not a drop ... has been added by India and other developing countries to 'the pool of world knowledge,", (H. Narain Curr. Sci. 65, 739-742; 1993).

I believe that nothing much has changed during the intervening years.
Why cannot India and China, two Asian giants, break the mediocrity barrier, despite their strong fundamentals?

Culture is a major impediment, as Poo points out. Although India is a democracy, the Asian tradition of respecting authority and hierarchy remains strong in our society, spawning conformity and nepotism. Creativity in science can be fostered only in a free and unfettered intellectual environment.

Cultural shifts take time. Meanwhile, I believe structural changes can help these fast-developing Asian countries to achieve more in terms of high-impact scientific research, whatever the level of investment.

Starting with universities and other centres of higher learning, such measures should include introducing innovative courses in basic sciences at post-graduate levels (in India, the institutes of technology are a good place to start) and fostering better links between research organizations and industries.

C. P. Rajendran

Centre for Earth Science Studies, Akkulam, Trivandrum 695031, India

\section{Ecological and political costs of river diversion}

Sir - In the Commentary "Agriculture of the future" (Nature 428, 215; 2004), T. C. Tso discusses the problems faced by China's agricultural sector and suggests several possible approaches to the problem of feeding the world's largest population. We support many of the suggested solutions, such as reducing farmers' costs, providing more funds for agricultural research and adopting new techniques to improve agricultural products.

However, we strongly oppose Tso's proposal to divert water from southwest China to its northern and eastern areas, especially the idea of diverting water from the 'big U-turn' of the Yarlung Zangbo river in Tibet.

The 'big U-turn' is located in the geologically fragile region of the Himalaya mountains where the Indian Plate meets the Eurasian Plate. The problems of geologic instability and frequent landslides alone are enough to make any huge hydraulic projects difficult or even impossible. Additionally, the Yarlung Zangbo is an international river, passing through China, India and Bangladesh. A diversion project will undoubtedly change the hydrological conditions, especially the volume of water available to the lower countries, and is likely to cause an international dispute. Moreover, the 'big U-turn' is located in one of the world's biodiversity hotspots. Tso's proposed water-diversion project would irreversibly damage the ecosystems of the world's deepest and longest canyon, particularly the aquatic fauna.

Finally, we believe that China's water problems would be best addressed by the adoption of sound water planning and management, water conservation and efficient irrigation techniques - not by large-scale diversions.

Anping Chen*, Changdu Chen $\dagger$

${ }^{*}$ Department of Ecology and Evolutionary Biology, Princeton University, Princeton,

New Jersey 08544-1003, USA

$\dagger$ Department of Ecology, Peking University, Beijing 100871, China

\section{Turing's war work counts for more than computers}

Sir - John L. Casti, in his fine review of Alan Turing: Life and Legacy of a Great Thinker, edited by Christof Teuscher ("Touring artificial minds" Nature 428, 258 ; 2004), proposes that Turing had more impact on everyday life than the man named by Time magazine as Person of the Century, Albert Einstein (Time 154, 27; 1999). Casti suggests that Turing's 1936 paper provided the "theoretical backbone" for all computers to come.

Although Turing, a hero of mine, certainly was one of the greatest, we should keep in mind that his paper essentially just elegantly rephrased Kurt Gödel's 1931 results and Alonzo Church's extension thereof. It did not have any impact on the construction of the first working programcontrolled computer. That was made in Berlin by Konrad Zuse in 1935-1941 and was driven by practical considerations, not theoretical ones.

In fact, the greatest impact that Alan Turing made on daily life was probably through his contribution to cracking the Enigma code, used by the German military during the Second World War, which is sometimes cited as a decisive event of the war.

Jürgen Schmidhuber

Dalle Molle Institute for Artificial Intelligence (IDSIA), Galleria 2, 6928 Manno-Lugano, Switzerland

\section{correspondence}

Contributions to Correspondence may be submitted to corres@nature.com. They should be no longer than 500 words, and ideally shorter. Published contributions are edited. 